\title{
Exact exchange for correlated electrons
}

\author{
P. Novák ${ }^{*}, 1$, J. Kuneš ${ }^{1,2}$, L. Chaput ${ }^{3}$, and W. E. Pickett ${ }^{2}$ \\ Institute of Physics of ASCR, Cukrovarnická 10, 16253 Prague 6, Czech Republic \\ 2 Department of Physics, University of California, Davis CA 95616, USA \\ ${ }^{3}$ Laboratoire de Physique des Matériaux, UMR 75560, ENSMN, Parc de Saurupt, 54042 Nancy, France
}

Received 19 October 2005, revised 16 November 2005, accepted 22 November 2005

Published online 10 January 2006

PACS 71.15.Mb, 71.27.+a

A density functional based "exact exchange for correlated electrons" (EECE) method of electronic structure determination, intended as an improved initial point for the description of systems containing correlated electrons, is proposed and its implementation is described. This approach bears strong resemblance to certain methods already in use: in implementation it is much like the LDA+U method with the important distinction that it is parameter-free; in concept has much in common with previous 'exact exchange' and self-interaction corrected approaches. The EECE method is applied to several systems with varying correlation strengths and characters $\left(\mathrm{FeF}_{2}, \mathrm{NiO}, \mathrm{hcp} \mathrm{Gd}, \mathrm{FeAl}\right)$ and the results are compared with those obtained by LDA +U and Hartree-Fock methods as well as results within the local spin density and generalized gradient approximations to density functional theory.

(C) 2006 WILEY-VCH Verlag GmbH \& Co. KGaA, Weinheim

\section{Introduction}

Density functional theory (DFT) provides the basis for most calculations of the electronic structure of solids [1], but the approximations used at present - local spin density approximation (LSDA) and generalized gradient approximation (GGA) - have well known shortcomings when applied to systems with a strong electron correlation. In many cases an incorrect character of the ground state is obtained, and in partially-filled narrow band systems bands are improperly clustered around the Fermi level. The most common viewpoint of these difficulties is that the main problem is not the DFT correlation functional per se but rather the local and very approximate treatment of the exchange energy. Several partial remedies, which might be classified as "correlated band theories," are in use. Most of these are formulated in terms of a correction to the LSDA density functional in favor of an orbital-dependent functional, and result in a generalization of the Kohn-Sham procedure for obtaining the minimum of the functional and leads to some type of correlated band structure.

The most thoroughly tested of these correlated band theories is the LDA+U approach [2], which has been built into several widely used codes. Its behavior is relatively well understood, although the interplay of the correlation effects (which tends to localize orbitals in some sense) and mixing with ligands (which tends to promote itineracy) still produces some surprises [3]. The LDA+U method is no longer ab-initio: a choice has to be made of which orbitals to treat as correlated, the Coulomb repulsion strength (Hubbard parameter) $U$ and the exchange constant $J$ must be inserted, and a few differing LDA+U versions are in use so the method is not even unique (see the paper of Petukhov et al. [4] for discussion).

\footnotetext{
Corresponding author: e-mail: novakp@fzu.cz
} 
Applying the Hartree-Fock (HF) method would seem to be a natural improvement, since by fiat it includes exchange exactly and it is self-interaction free. Unfortunately, the HF method is difficult to apply to periodic solids due to the strong nonlocal nature of the exchange potential, and it is known to present difficult problems when approaching the weakly correlated regime [5]. Unless the exchange interaction is screened in some manner, HF also overestimates corrections even in the strongly correlated regime. The HF method has been implemented by a few groups, for example in the CRYSTAL code, newer versions of which also have the DFT based approximations as an option [8]. In many ways HF is inferior for solids in several respects compared to LSDA and GGA but it has some advantages. The approach is transparent (minimizing the energy of a determinant wavefunction) and to extend it to include correlation seems straightforward, but becomes tedious.

Recently 'hybrid density functionals' have gained visibility [9], with the most used version being the B3LYP functional [10]. These hybrid functionals begin with the DFT approach, but to address the problems noted above, they mix the exchange part of the approximate DFT functional with the HF exact exchange, and use also a nonlocal correlation functional. The results obtained recently for crystals have been encouraging $[11,12]$.

Another approach is the self-interaction-corrected (SIC) local density approximation as applied to solids [13]. The SIC method appends a correction to the LSDA functional which removes the selfHartree and local self-exchange and self-correlation energies from orbitals which become localized (selfconsistently) due to the correction. This approach has been applied to a considerable number of systems, and seems to be realistic in producing localization of correlated orbitals and giving much improved energetics compared to LSDA. The eigenvalues of the correlated orbitals do not however bear any close relation to observed single particle binding energies.

In the present paper we propose an alternative approach to those mentioned above. The overarching concept is to retain the LSDA (or GGA) description of uncorrelated states, where it works very well, but to perform exact exchange on the correlated electrons (EECE), removing the local treatment they are given in LSDA (including the self-interaction). Processes involving the interaction between correlated and uncorrelated orbitals are also included within LSDA, since such processes are normally not involved in the problematic behavior.

In the following sections we describe its implementation in the widely used WIEN2k package [14] and apply the approach to several correlated materials to assess its performance and utility. WIEN2k is based on the full potential linearized augmented plane wave method (FPLAPW), an approach which belongs to the most precise and reliable ways to calculate the electronic structure of solids. In the FPLAPW method the crystal is divided into non-overlapping atomic spheres and the interstitial region.

The EECE treatment parallels that of LDA+U in the following ways:

- the subspace $S_{\text {corr }}$ spanned by states of correlated electrons (usually d states of transition metal atoms or f states of rare earths or actinides) is identified as well as its projection operators.

- parts of the exchange-correlation energy and potential from the density functional theory (DFT) expressions that corresponds to $S_{\text {corr }}$ subspace are removed. In the LDA+U method this is the 'double counting' term.

- a new functional that presumably better reflects the strong correlation is inserted. In the LDA+U method this term has the HF form, with correlation taken into account by screening the $U$ and $J$ parameters. The corresponding potential term is nonlocal (orbitally dependent).

The second bullet is the most problematic part of approaches attempting to treat on-site correlations explicitly. By the nature of Kohn-Sham DFT theory the exchange-correlation functional is a non-linear functional of the total density. While one can certainly partition the density in a well defined way, e.g. 'correlated' and 'uncorrelated' orbitals, corresponding partitioning of the exchange-correlation energy, i.e. correlated, uncorrelated and cross term, is not unique and thus remains to be specified. One is typically guided by certain limits and by the desired performance of the method. As examples we mention the fully localized limit LDA+U method (atomic limit) [6], the around-mean-field LDA+U (uniform occupation limit) [7], or self-interaction correction (SIC) (single electron limit) [15]. In the following sections we propose a specific double counting scheme which is analogous to that in the SIC method in 
the sense that we identify a specific part of the density and subtract the corresponding exchangecorrelation energy. In the limit of non-overlapping correlated orbitals only, the present method reduces to the Hartree-Fock method.

\section{Density functional and total energy formula}

Building on the ideas discussed in the Introduction, we consider the functional obtained from the LDA (or GGA) functional by removing the local exchange-correlation energy of the correlated orbitals, and replacing it with the HF exchange energy $E_{\mathrm{X}}^{\mathrm{HF}}$ of these orbitals. Specifically, we define the EECE functional as

$$
E\left[\rho,\left\{\phi_{m}\right\}\right]=E^{\mathrm{LDA}}[\rho]+E_{\mathrm{X}}^{\mathrm{HF}}\left[n^{\sigma \sigma^{\prime}}(\rho) ;\left\{\phi_{m}\right\}\right]-E_{\mathrm{dc}}\left[n^{\sigma \sigma^{\prime}}(\rho) ;\left\{\phi_{m}\right\}\right] .
$$

Note that exchange (and correlation) between correlated and uncorrelated orbitals are treated within LDA (GGA), since LDA is expected to be appropriate for these interactions. Only exchange within the correlated subshell on each atom is treated by the exact (HF) expression. The second and third terms are explicit functionals of the occupation matrix $n^{\sigma \sigma^{\prime}}$, which in turn depends on the total density $\rho(\boldsymbol{r})$. We have indicated explicitly the parametric dependence of these terms on the correlated orbitals $\phi_{m}$, which have to be specified beforehand. It should be pointed out that for the purpose of definition of the functional the correlated orbitals are fixed, i.e. the correlated density is uniquely determined by the corresponding occupation matrix, and variation of the functional is carried out only with respect to the KohnSham orbitals that serve to represent the density. In practical calculations the correlated orbitals may be allowed to relax (as they are in our implementation), however this relaxation does not have variational character.

In this EECE functional $E^{\mathrm{LDA}}$ stands for any semilocal DFT functional (GGA for example), and the intra-atomic HF-like exchange $E_{\mathrm{X}}^{\mathrm{HF}}$ and the double-counting correction $E_{\mathrm{dc}}$ are given explicitly by

$$
\begin{aligned}
& E_{\mathrm{X}}^{\mathrm{HF}}\left[n^{\sigma \sigma^{\prime}}(\rho) ;\left\{\phi_{m}\right\}\right]=-\frac{1}{2} \sum_{m_{1} \cdot m_{4}}^{\sigma, \sigma^{\prime}} n_{m_{1} m_{2}}^{\sigma \sigma^{\prime}}\left\langle m_{1}, m_{3}\left|V^{e e}\right| m_{4}, m_{2}\right\rangle n_{m_{3} m_{4}}^{\sigma^{\prime} \sigma}, \\
& E_{\mathrm{dc}}\left[n^{\sigma \sigma^{\prime}}(\rho) ;\left\{\phi_{m}\right\}\right]=E_{\mathrm{XC}}^{\mathrm{LDA}}\left[\rho_{\mathrm{corr}}\right],
\end{aligned}
$$

$n_{m_{1} m_{2}}^{\sigma \sigma^{\prime}}$ are elements (4) of the occupation matrix and $E_{\mathrm{XC}}^{\mathrm{LDA}}\left[\rho_{\text {corr }}\right]$ is the exchange-correlation energy evaluated with the correlated density. Note that this HF functional includes the diagonal term, so it subtracts off the self-interaction of the correlated orbitals that has been included in the Hartree energy. Note also that the correlation energy corresponding to $\rho_{\text {corr }}$ has been subtracted out, not because it is treated better in another term (there is no such term in this EECE functional) but simply because local correlation is inappropriate for correlated orbitals.

The occupation matrix is obtained by projection of the occupied Kohn-Sham states $\left|\varphi_{k}\right\rangle$ onto the predefined orbitals:

$$
n_{m m^{\prime}}^{\sigma \sigma^{\prime}}=\sum_{\varepsilon_{k} \leq E_{F}}\left\langle\varphi_{k} \mid \phi_{m}^{\sigma}\right\rangle\left\langle\phi_{m^{\prime}}^{\sigma^{\prime}} \mid \varphi_{k}\right\rangle .
$$

The corresponding correlated density is obtained from

$$
\rho_{\text {corr }}^{\sigma \sigma^{\prime}}(\boldsymbol{r})=\sum_{m, m^{\prime}} \phi_{m}^{\sigma *}(\boldsymbol{r}) n_{m m^{\prime}}^{\sigma \sigma^{\prime}} \phi_{m^{\prime}}^{\sigma^{\prime}}(\boldsymbol{r}) \text {. }
$$

Using the Kohn-Sham ansatz the following equations are obtained by variation of the functional (1) with respect to $\varphi_{k}^{*}(\boldsymbol{r})$ :

$$
H^{\mathrm{LDA}} \varphi_{k}^{\sigma}(\boldsymbol{r})+\sum_{m \sigma, m^{\prime} \sigma^{\prime}}\left[\frac{\partial E_{\mathrm{X}}^{\mathrm{HF}}}{\partial n_{m m^{\prime}}^{\sigma \sigma^{\prime}}} \frac{\partial n_{m m^{\prime}}^{\sigma \sigma^{\prime}}}{\partial \varphi_{k}^{\sigma^{*}}(\boldsymbol{r})}-\int \mathrm{d} \boldsymbol{r}^{\prime} \frac{\partial E_{\mathrm{XC}}^{\mathrm{LDA}}}{\partial \rho_{\mathrm{corr}}^{\sigma \sigma^{\prime}}\left(\boldsymbol{r}^{\prime}\right)} \frac{\partial \rho_{\mathrm{corr}}^{\sigma \sigma^{\prime}}\left(\boldsymbol{r}^{\prime}\right)}{\partial n_{m m^{\prime}}^{\sigma \sigma^{\prime}}} \frac{n_{m m^{\prime}}^{\sigma \sigma^{\prime}}}{\varphi_{k}^{\sigma}(\boldsymbol{r})^{*}}\right]=\varepsilon_{k}^{\sigma} \varphi_{k}^{\sigma}(\boldsymbol{r})
$$


$H^{\mathrm{LDA}}$ is the usual effective LDA/GGA Hamiltonian, and $\varphi_{k}$ are the Kohn-Sham wavefunctions. Using Eqs. (4) and (5) one can evaluate the functional derivatives on the right hand side explicitly:

$$
\begin{gathered}
\frac{\partial n_{m m^{\prime}}^{\sigma \sigma^{\prime}}}{\partial \varphi_{k}^{*}(\boldsymbol{r})}=\phi_{m}^{\sigma}(\boldsymbol{r})\left\langle\phi_{m^{\prime}}^{\sigma^{\prime}} \mid \varphi_{\boldsymbol{k}}\right\rangle, \\
\frac{\partial \rho_{\mathrm{corr}}^{\sigma \sigma^{\prime}}(\boldsymbol{r})}{\partial n_{m m^{\prime}}^{\sigma \sigma^{\prime}}}=\phi_{m}^{\sigma *}(\boldsymbol{r}) \phi_{m^{\prime}}^{\sigma^{\prime}}(\boldsymbol{r}) .
\end{gathered}
$$

We point out that although the double counting term $E_{\mathrm{dc}}$ has the same form as the LDA exchangecorrelation functional, it gives rise to a non-local potential in the Kohn-Sham equation because the argument is not the full density. Using Eqs. (7) and (8) the Kohn-Sham equations can be written as an eigenvalue problem with an additional non-local potential

$$
\begin{aligned}
& H^{\mathrm{LDA}} \varphi_{k}^{\sigma}+\sum_{m, m^{\prime}}\left|\phi_{m}^{\sigma}\right\rangle V_{m m^{\prime}}^{x}\left\langle\phi_{m^{\prime}}^{\sigma}\left|\varphi_{k}^{\sigma}-\sum_{m, m^{\prime}}\right| \phi_{m}^{\sigma}\right\rangle V_{m m^{\prime}}^{\mathrm{dc}}\left\langle\phi_{m^{\prime}}^{\sigma}\right| \varphi_{k}^{\sigma}=\varepsilon_{k}^{\sigma} \varphi_{k}^{\sigma}, \\
& V_{m m^{\prime}}^{x}=-\sum_{m_{3}, m_{4}} n_{m_{3}, m_{4}}^{\sigma \sigma}\left\langle m, m_{3}\left|V^{e e}\right| m_{4}, m^{\prime}\right\rangle, \\
& V_{m m^{\prime}}^{\mathrm{dc}}=\int \mathrm{d} \boldsymbol{r} V_{\mathrm{XC}}^{\mathrm{LDA}}\left(\rho^{\mathrm{corr}} ; \boldsymbol{r}\right) \phi_{m}^{\sigma *}(\boldsymbol{r}) \phi_{m^{\prime}}^{\sigma}(\boldsymbol{r}) .
\end{aligned}
$$

Derivation of the total energy formula is analogous to the standard Kohn-Sham procedure. The only term which cannot be evaluated explicitly from the density or occupation matrix, the Kohn-Sham kinetic energy, is expressed through the eigenvalue sum [16]. This brings in additional terms, consisting of the correction for double counting of the HF exchange energy in the eigenvalue sum, and the usual "miscounting" correction of the LDA XC energy:

$$
E_{\mathrm{tot}}=E_{\mathrm{LDA}}-\frac{1}{2} \operatorname{Tr}\left(V^{x} \hat{n}^{\dagger}\right)+E_{\mathrm{XC}}^{\mathrm{LDA}}\left[\rho^{\mathrm{corr}}\right]-\operatorname{Tr}\left(V^{d c} \hat{n}^{\dagger}\right) .
$$

Here $E_{\mathrm{LDA}}$ is the total energy formula for the local density functional including the eigenvalue sum. The traces are taken over the index labeling the correlated orbitals $\phi_{m}$. Evaluating the trace in the last term one arrives at an alternative expression

$$
\operatorname{Tr}\left(V^{\mathrm{dc}} \hat{n}^{\dagger}\right)=\int \mathrm{d} \boldsymbol{r} V_{\mathrm{XC}}^{\mathrm{LDA}}\left(\rho^{\mathrm{corr}} ; \boldsymbol{r}\right) \rho^{\mathrm{corr}}(\boldsymbol{r}) .
$$

\section{Implementation in the LAPW method}

The LAPW method is based on partitioning the space into atomic spheres and the interstitial region. It is natural, and in fact commonly done in current LAPW implementations of LDA+U method, to use this partitioning to define the correlated orbitals. The subspace of correlated orbitals is specified by angular momentum $l$ and projection onto a given atomic sphere. The $z$-axis projection of the angular momentum $(m)$ and the $z$ component of the spin $(\sigma)$ index the correlated orbitals. In the LAPW method there is some freedom in the radial extent of the orbitals. When constructing the occupation matrix we take this freedom into account simply by accumulating all radial contributions, i.e. the radial part of the projection operator is unity.

In the applications that we discuss in the following section, we make a minor approximation when constructing the correlated density using the average radial function described below. The correlated density (5) is written as

$$
\rho^{\mathrm{corr}}(\boldsymbol{r})=\sum_{m, m^{\prime}} n_{m m^{\prime}} \chi^{2}(r) Y_{l m}^{*}(\hat{r}) Y_{l m^{\prime}}(\hat{r})
$$




$$
\rho^{\mathrm{corr}}(\boldsymbol{r})=\sum_{L=0}^{2 l} \sum_{M=-L}^{L} \rho_{L M}^{\mathrm{corr}}(r) Z_{L M}(\hat{r}),
$$

where the product of spherical harmonics $Y_{l m}$ is replaced by a sum over real harmonics $Z_{L M}$ and the radial distribution is described by a single function $\chi(r)$. The spin indices were dropped for simplicity. The radial function is defined such that it reproduces exactly the spherical term in correlated density expansion

$$
\chi^{2}(r)=\frac{\rho_{00}^{\text {corr }}(r)}{N_{\text {corr }}} .
$$

The $N_{\text {corr }}$ factor, which is the total occupation of the correlated orbitals, insures normalization of the radial function. This minor approximation can readily be abandoned if it becomes of interest to allow the various radial functions to become distinct.

The matrix elements of the $V^{\mathrm{dc}}$ potential are calculated by expanding $V_{\mathrm{xc}}\left(\rho^{\text {corr }}\right)$ in the real harmonics basis (analogously to Eq. (13)) and evaluating the corresponding radial and spherical harmonics integrals. The matrix elements of the exchange potential are calculated using the standard expansion in terms of Slater integrals, which are evaluated with the radial function $\chi(r)$. Much of this formalism (and code) is almost identical to the implementation of the $\mathrm{LDA}+\mathrm{U}$ method, but now the Slater integrals are calculated explicitly rather than using parameterized screened values.

\section{Applications to selected materials}

Below the EECE method is applied to four systems with different degree and character of correlation: the correlated insulators $\mathrm{FeF}_{2}$ and $\mathrm{NiO}$, and the metals hcp $\mathrm{Gd}$ and $\mathrm{FeAl}$. The results are compared with corresponding results from LSDA, GGA, LDA+U and, when available, HF calculations. As a starting point for these EECE calculations the selfconsistent LSDA density and occupation matrix was adopted. All calculations were spin polarized, with ferromagnetic spin configuration for hcp Gd and FeAl and antiferromagnetic for $\mathrm{FeF}_{2}$ and $\mathrm{NiO}$. The magnetic moments and density of states were evaluated for the experimental crystal structure, while the equilibrium volume and the bulk modulus were determined from the dependence of the total energy on the unit cell volume (for $\mathrm{FeF}_{2}$ and hcp Gd the ratio cla was fixed at its experimental value).

In the LAPW method there are two important parameters determining the precision of the calculation: the number $N_{k}$ of $k$ points in the Brillouin zone and the number $N_{b}$ of basis functions (which may vary slightly with the $k$ point). In addition for present calculations the radius of the cation atomic sphere must be considered, as only within the sphere the EECE and LDA+U potentials are considered. The LDA+U calculations require as input the Hubbard parameter $U$ and the exchange parameter $J$. All these quantities are summarized in Table 1. The 'fully localized limit' (FLL) version of $\mathrm{LDA}+\mathrm{U}$ was employed for $\mathrm{FeF}_{2}$, $\mathrm{NiO}$, hep Gd [6] while the 'around mean field' (AMF) version [7] was used for FeAl as it leads to a correct nonmagnetic state for this compound.

Table 1 For each of the systems considered here, this table provides the number of $k$-points $N_{k}$, the number of the basis functions $N_{\mathrm{b}}$, the radius of the cation atomic sphere $R_{\mathrm{s}}$ (a.u.), the Hubbard interaction strength $U$ and exchange constant $J(\mathrm{eV})$.

\begin{tabular}{llllll}
\hline & $N_{k}$ & $N_{\mathrm{b}}$ & $R_{\mathrm{s}}$ & $U$ & $J$ \\
\hline $\mathrm{FeF}_{2}$ & 1000 & 570 & 1.9 & 6 & 0.95 \\
$\mathrm{NiO}$ & 600 & 300 & 2 & 8 & 0.95 \\
hcp Gd & 2000 & 150 & 3 & 7.65 & 0.7 \\
FeAl & 1000 & 160 & 2 & 8.7 & 0.95 \\
\hline
\end{tabular}


Table 2 Properties of $\mathrm{FeF}_{2}$ calculated by several methods. $\Delta_{\mathrm{dd}}$ is the splitting between the highest occupied and the lowest empty $3 \mathrm{~d}$ state of Fe. The HF value of $\Delta_{\mathrm{dd}}$ was estimated from Fig. 2 of Ref. [17].

\begin{tabular}{lllllll}
\hline & LSDA & GGA & LDA+U & EECE & HF & exp. \\
\hline$V_{0}\left(\mathrm{~nm}^{3}\right)$ & 0.0676 & 0.0747 & 0.0694 & 0.0744 & 0.0781 & 0.0727 \\
$B(\mathrm{GPa})$ & 133 & 104 & 131 & 112 & 106 & 100 \\
$\mathrm{Gap}(\mathrm{eV})$ & 0 & 0.35 & 3.0 & 4.2 & & \\
$\Delta_{\mathrm{dd}}(\mathrm{eV})$ & 1.27 & 2.11 & 6.55 & 17.77 & 21 & \\
$m_{\mathrm{Fe}}\left(\mu_{\mathrm{B}}\right)$ & 3.47 & 3.50 & 3.72 & 3.67 & 3.93 & 3.75 \\
\hline
\end{tabular}

\subsection{Iron difluoride}

$\mathrm{FeF}_{2}$ crystallize in the rutile-type structure, where each $\mathrm{Fe}$ atom is octahedrally coordinated by the $\mathrm{F}$ ligands. $\mathrm{FeF}_{2}$ is an ionic compound with $\mathrm{Fe}^{2+}\left(3 \mathrm{~d}^{6}\right)$ ions in a high spin state. HF calculations using the program CRYSTAL were performed by Valerio et al. [17]. $\mathrm{FeF}_{2}$ is predicted to be metallic by LSDA (the standard problem of the Mott insulator in LSDA), a small gap is obtained in the GGA calculation as allowed by the crystal field, while LDA+U leads to a large, and reasonable, gap of $3 \mathrm{eV}$ (Table 2). The lowest unoccupied band as given by LDA $+\mathrm{U}$ has minority spin Fe $3 \mathrm{~d}_{x z}$ character. The DOS obtained by EECE is very different, with the Hubbard splitting being strongly overestimated as in HF calculations. We characterize this splitting by the energy difference $\Delta_{\mathrm{dd}}$ between maxima of DOS of the highest occupied and the lowest unoccupied $\mathrm{Fe}(3 \mathrm{~d})$ states. $\Delta_{\mathrm{dd}}(\mathrm{EECE})$ is almost three times larger than $\Delta_{\mathrm{dd}}(\mathrm{LDA}+\mathrm{U})$ and it is close to its HF value. The density of states projected on the iron ion as obtained by EECE method is displayed in Fig. 1, where the positions of the peaks in the DOS calculated by HF method [17] are also indicated. The EECE gap is much smaller than $\Delta_{\mathrm{dd}}$, with the lowest unoccupied states having Fe and $\mathrm{F} s$ character. In Table 2 the calculated quantities are summarized and compared with their experimental values; note that the volume is much improved over both the LDA and HF values. It can be observed that the spin magnetic moment $m_{s}$ increases in the sequence LSDA $<$ GGA $<$ LDA $+\mathrm{U}<\mathrm{HF}$, indicating increasing localization of electrons in the $3 \mathrm{~d}$ majority spin states of $\mathrm{Fe}^{2+}$. The EECE value is much larger than the one of LSDA and GGA, but smaller comparing to the HF result.

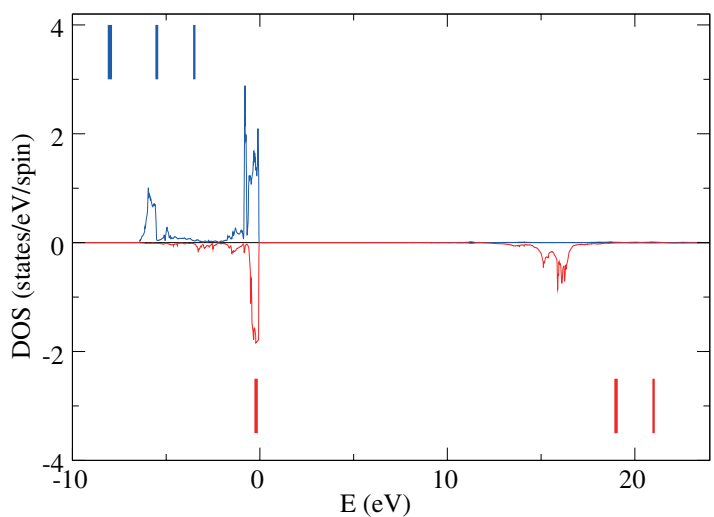

Fig. 1 (online colour at: www.pss-b.com) Results for $\mathrm{FeF}_{2}$, from the EECE method, of the Fe $3 \mathrm{~d}$ density of states for majority (positive) and minority (negative) spin directions. Vertical bars indicate the position of analogous peaks in the HF calculation of Valerio et al. [17]. The top of the valence band defines the zero of energy.

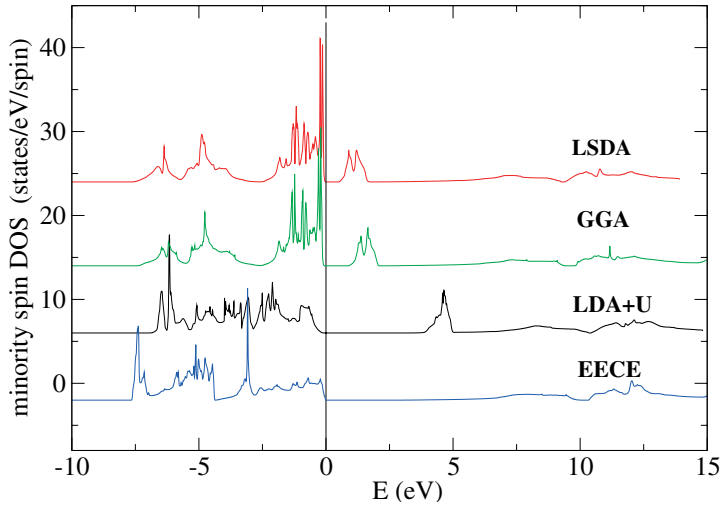

Fig. 2 (online colour at: www.pss-b.com) Density of the minority spin states in $\mathrm{NiO}$, calculated by LSDA, GGA, LDA+U and EECE methods. The plots are displaced vertically for clarity; the value of each curve is zero at $-10 \mathrm{eV}$. The $\mathrm{LDA}+\mathrm{U}$ and EECE results are distinguished not only by a widening of the $\mathrm{d}-\mathrm{d}$ (Mott) gap, but also by increased mixing of the occupied $3 \mathrm{~d}$ orbitals with the oxygen $2 p$ bands. 


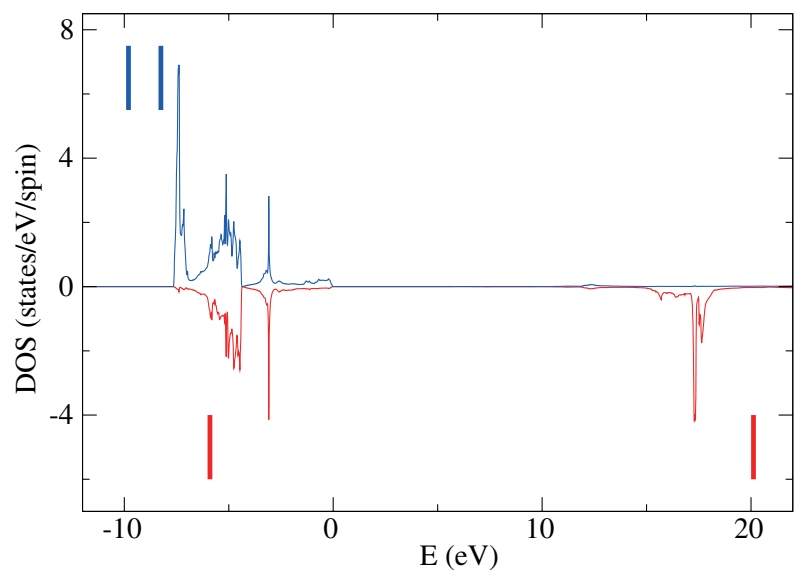

Fig. 3 (online colour at: www.pss-b.com) $\mathrm{NiO}$ :DOS projected on the majority spin states (positive) and minority spin states (negative) of the $\mathrm{Ni}$ atom. Vertical bars indicate the position of peaks in the DOS calculated by HF method. The Fermi energy is at $E=0$.

\subsection{Nickel oxide}

The DOS of minority spin states calculated by LSDA, GGA, LDA+U and EECE is shown in Fig. 2. In Fig. 3 the EECE density of states projected onto majority and minority spin states of $\mathrm{Ni}$ are displayed. To indicate how the eigenvalues compare with the HF results, the peaks in analogous HF DOS are also indicated. The HF calculation was performed by Towler et al. [18] using the program CRYSTAL. Table 3 summarizes the calculated quantities. Contrasts between EECE and HF become interesting: the volumes and $\Delta_{\mathrm{dd}}$ separations are similar as might be expected, but the calculated magnetic moments differ by more than $10 \%$.

\section{3 hep Gd}

Gadolinium has a half filled $4 \mathrm{f}$ shell, and we take the $4 \mathrm{f}$ as correlated to apply the present method. To our knowledge no HF calculation of the Gd electronic structure exists, but EECE is compared with LSDA, GGA and LDA+U. In all cases the $4 \mathrm{f}$ states are included among the valence states. In the LDA+U calculation the value of $U=7.65 \mathrm{eV}$ was chosen to reproduce the experimentally observed splitting $12 \mathrm{eV}$ between majority and minority $4 \mathrm{f}$ spin states of $\mathrm{Gd}$. This value is comparable to, but larger than, the value $U=6.7 \mathrm{eV}$ used in Ref. [21]. The results are summarized in Table 4. The total density of majority and minority states as calculated by the four methods is shown in Fig. 4. Remarkably, the energy difference between the occupied and unoccupied $4 \mathrm{f}$ states calculated by the EECE is $12.68 \mathrm{eV}$, close to the $\mathrm{LDA}+\mathrm{U}$ and the experimental values.

Table 3 Properties of NiO calculated by several methods. $\Delta_{\mathrm{dd}}$ is the splitting between occupied and empty Ni $3 d$ minority spin states. The HF value of $\Delta_{\mathrm{dd}}$ was estimated from Fig. 5(e) of Ref. [18]. Experimental data for magnetic moments [20] were measured at $300 \mathrm{~K}$.

\begin{tabular}{lllllll}
\hline & LSDA & GGA & LDA+U & EECE & HF & exp. \\
\hline$V_{0}\left(\mathrm{~nm}^{3}\right)$ & 0.0338 & 0.0373 & 0.0353 & 0.0386 & 0.0390 & 0.0369 \\
$B(\mathrm{GPa})$ & 254 & 191 & 192 & 206 & 145 & 214 \\
gap $(\mathrm{eV})$ & 0.42 & 0.96 & 3.14 & 3.0 & & 4.2 \\
$\Delta_{\mathrm{dd}}(\mathrm{eV})$ & 1.6 & 2.1 & 7.4 & 25.9 & 25.0 & \\
$m_{\mathrm{Ni}}\left(\mu_{\mathrm{B}}\right)$ & 1.20 & 1.38 & 1.73 & 1.71 & 1.92 & $1.90(20)$ \\
\hline
\end{tabular}




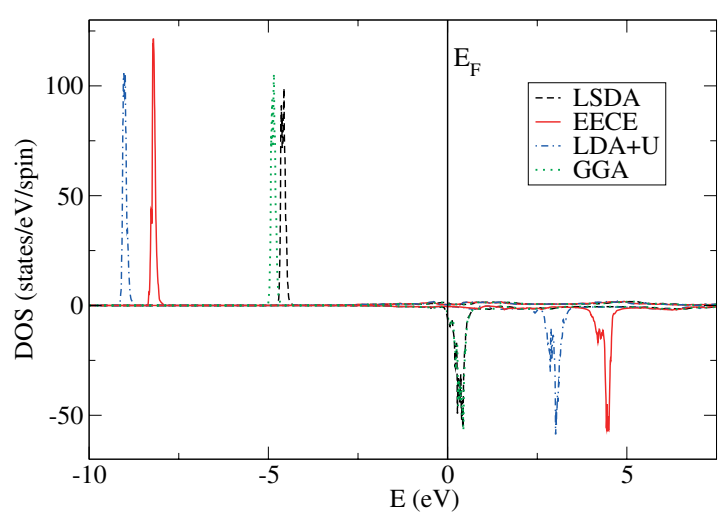

Fig. 4 (online colour at: www.pss-b.com) hep Gd Total density of states for majority (positive) and minority (negative) spin states.

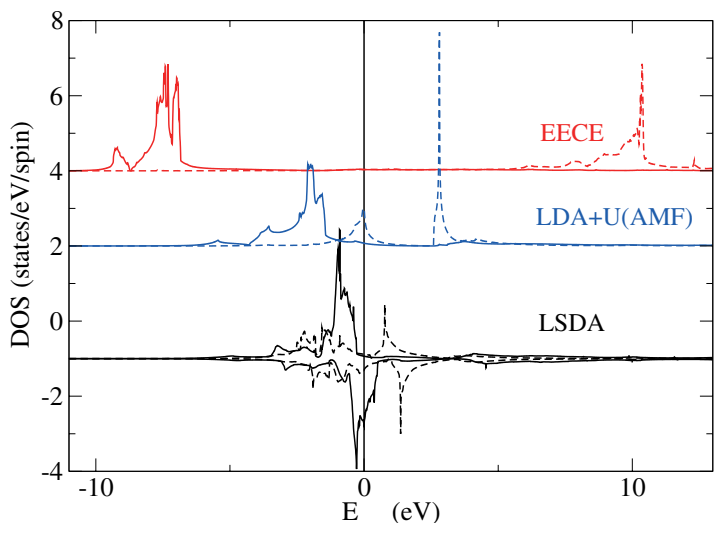

Fig. 5 (online colour at: www.pss-b.com) FeAl. DOS projected on the $t_{2 \mathrm{~g}}$ (full curves) and $e_{\mathrm{g}}$ (dashed curves) states of the iron atom as calculated by different methods. LSDA results in a magnetic solution, thus the DOS corresponding to both majority (positive) and minority (negative) states are displayed. EECE and LDA+U(AMF) give a nonmagnetic ground state with identical DOS for spin-up and spin-down spin states.

\section{4 $\mathrm{FeAl}$}

$\mathrm{FeAl}$ is a nonmagnetic metal (and thus presumably rather weakly correlated at most), but both LSDA and GGA predict the ground state to be ferromagnetic. An LDA $+\mathrm{U}$ calculation gave a nonmagnetic ground state, but required a rather large value of $U \geq 3.7 \mathrm{eV}$ and the AMF version of LDA+U had to be employed [23]. The HF method is known to yield unphysical picture when applied to metals (e.g. vanishing DOS at the Fermi energy) [5]. As seen from the results obtained for $\mathrm{FeF}_{2}$ and NiO, EECE is similar to HF. Still, it is instructive to perform the EECE calculation for FeAl and we applied it to the iron $3 \mathrm{~d}$ states. The selfconsistent procedure converges to the nonmagnetic state with strong $\mathrm{Fe}(3 \mathrm{~d})$ electron localization: the $e_{\mathrm{g}}$ states are empty, while $t_{2 \mathrm{~g}}$ states are fully occupied, which situation brings to mind a 'strong crystal field' picture but is really just a correlation gap effect. The system remains metallic due to $\mathrm{Fe} 3 \mathrm{p}$ and $4 \mathrm{~s}$ states and $\mathrm{Al} 3 \mathrm{p}$ states at the Fermi level. However, the Fe $3 \mathrm{~d}$ states become so localized that they no longer participate in the chemical bonding. As a result the equilibrium volume is far too large. The densities of states calculated by several methods and projected onto $t_{2 \mathrm{~g}}$ and $e_{\mathrm{g}}$ states of Fe are displayed in Fig. 5.

\section{Discussion}

The results obtained for $\mathrm{FeF}_{2}$ and $\mathrm{NiO}$ show that for the strongly correlated electrons in these compounds, EECE gives results very similar to HF. It is recognized that the HF method predicts occupied

Table 4 Properties of hcp Gd calculated by several methods. $V_{0}$ and $B$ denote the equilibrium volume and the bulk modulus, $\Delta_{\mathrm{f}}$ is the splitting between majority and minority $4 \mathrm{f}$ states. The experimental value of of the spin moment $m$ was taken from Ref. [22].

\begin{tabular}{llllll}
\hline & LSDA & GGA & LDA+U & EECE & exp. \\
\hline$V_{0}\left(\mathrm{~nm}^{3}\right)$ & 0.0585 & 0.0673 & 0.0607 & 0.0700 & 0.0663 \\
$B(\mathrm{GPa})$ & 24.9 & 35.6 & 40.3 & 50.4 & 38.0 \\
$\Delta_{\mathrm{f}}(\mathrm{eV})$ & 4.78 & 5.28 & 12.0 & 12.68 & 12.0 \\
$m_{\mathrm{Gd}}\left(\mu_{\mathrm{B}}\right)$ & 7.36 & 7.57 & 7.82 & 7.44 & 7.63 \\
\hline
\end{tabular}


Table 5 Equilibrium volume, bulk modulus, and $\mathrm{Fe}$ moment in $\mathrm{FeAl}$ calculated by several methods.

\begin{tabular}{llllll}
\hline & LSDA & GGA & LDA+U & EECE & exp. \\
\hline$V_{0}\left(\mathrm{~nm}^{3}\right)$ & 0.0226 & 0.0239 & 0.0236 & 0.0343 & 0.0249 \\
$B(\mathrm{GPa})$ & 221 & 181 & 162 & 65 & 150 \\
$m_{\mathrm{Fe}}\left(\mu_{\mathrm{B}}\right)$ & 0.78 & 0.77 & 0 & 0 & 0
\end{tabular}

electron states to be too localized and therefore greatly overestimates the gap, and a similar tendency is seen in the EECE calculations. The splitting $\Delta_{\mathrm{dd}}$ between occupied and empty $3 \mathrm{~d}$ minority spin states is an order of magnitude larger than the one calculated by LSDA and GGA (Tables 2, 3). The energies of the states to which EECE is not applied (the uncorrelated states) remain essentially unchanged; for $\mathrm{NiO}$ this effect may be seen in Fig. 2. In FeAl the electron band structure of the Fe $3 \mathrm{~d}$ states is particularly strongly affected - contrary to LSDA and LDA+U these states do not appear at the Fermi level, the occupied levels being $8 \mathrm{eV}$ below and unoccupied $11 \mathrm{eV}$ above $E_{\mathrm{F}}$.

More encouraging is the case of hcp Gd where the splitting between occupied and empty $4 \mathrm{f}$ states is in reasonable agreement with the experiment and the DOS is close to the one calculated by LDA+U, where however $U$ was chosen to fit the splitting. In both $\mathrm{FeF}_{2}$ and $\mathrm{NiO}$ the value of the spin magnetic moment becomes larger in sequence LSDA $<$ GGA $<$ EECE $<$ HF indicating that the localization of the magnetic electrons also increases along this sequence. For NiO, where both spin and orbital parts of the magnetic moment were determined experimentally, EECE gives better agreement than LSDA and GGA (see Table 3).

The equilibrium volume is in all cases overestimated in the EECE calculation, contrary to LSDA which underestimates it. Clearly corrections could be constructed that start with EECE and, by admixing to it correlation, would yield an equilibrium geometry closer to the experimental one. Implementing such an extension would be straightforward as the DFT correlation part of the energy functional and potential are calculated separately in the WIEN2k code. The most striking discrepancy for equilibrium volume is found in FeAl, where it is clearly caused by the fact that the $3 d$ electrons are too localized and thus do not contribute to the bonding.

The EECE method depends crucially on the Slater integrals. We thus summarize their values in the Table 6. It is seen that $F^{k}$ depend only slightly on the type of atom and the compound. An unappealing feature of the EECE implementation to FPLAPW method is that the results depend on the radius $R_{\mathrm{s}}$ of the atomic sphere, because the EECE potential is zero outside the spheres and also the Slater integrals slightly depend on $R_{\mathrm{s}}$. We have checked for $\mathrm{NiO}$ that this dependence is not dramatic and the results change smoothly and predictably. In fact by reducing $R_{\mathrm{s}}$, the region of space in which EECE operates is reduced, making the results become closer to LSDA and thus also to the experiment.

\section{Conclusions}

In this paper we have proposed an 'exact exchange for correlated electrons' method of electronic structure calculation, implemented it into the WIEN2k implementation of the LAPW method, and tested its predictions on four different types of $\mathrm{d}$ and $\mathrm{f}$ electron materials. The results obtained document that this EECE method gives results closely related to those obtained with the Hartree-Fock method. EECE is

Table 6 Slater integrals in $\mathrm{eV}$ for the transition metal atoms in the compounds considered.

\begin{tabular}{lllll}
\hline & $F^{0}$ & $F^{2}$ & $F^{4}$ & $F^{6}$ \\
\hline $\mathrm{Fe}: \mathrm{FeF}_{2}$ & 25.94 & 12.36 & 7.76 & 5.59 \\
$\mathrm{Ni}: \mathrm{NiO}$ & 27.46 & 12.74 & 7.92 & 5.68 \\
Gd:hcp Gd & 29.67 & 13.61 & 8.87 & 6.24 \\
Fe:FeAl & 25.16 & 11.84 & 7.40 & 5.31 \\
\hline
\end{tabular}


implemented in the WIEN2k code, based on the FLAPW approach to the density functional theory. It thus represents a counterpart to program CRYSTAL in which to originally HF code the local spin density and generalized gradient approximations were added. Due to the Gaussian basis used by CRYSTAL it is difficult to apply it to systems with strongly localized electrons. No such technical restriction applies to the present method. Moreover EECE is relatively transparent and it has very little additional requirements on computer time and memory. It is promising starting point for the further improvement of orbital-dependent density functionals.

Acknowledgments This work was supported by the project A1010214 of the Grant Agency of the AS CR. J.K. and W.E.P. were supported by U.S. Department of Energy grant DE-FG03-01ER45876 and DOE's Computational Materials Science Network.

\section{References}

[1] See R. O. Jones and O. Gunnarsson, Rev. Mod. Phys. 61, 689 (1989) for an extensive review.

[2] V. I. Anisimov, J. Zaanen, and O. K. Andersen, Phys. Rev. B 49, 943 (1991).

[3] K.-W. Lee, J. Kuneš, P. Novák, and W. E. Pickett, Phys. Rev. Lett. 94, 026403 (2005).

[4] A. G. Petukhov, I. I. Mazin, L. Chioncel, and A. I. Liechtenstein, Phys. Rev. B 67, 153106 (2003).

[5] G. D. Mahan, Many-Particle Physics (Plenum Press, New York, 1990).

[6] V. I. Anisimov, I. V. Solovyev, M. A. Korotin, M. T. Czyzyk, and G. A. Sawatzky, Phys. Rev. B 48, 16929 (1993).

[7] M. T. Czyzyk and G. A. Sawatzky, Phys. Rev. B 49, 14211 (1994).

[8] V. R. Saunders, R. Dovesi, C. Roetti, R. Orlando, C. M. Zicovich-Wilson, N. M. Harrison, K. Doll, B. Civalleri, I. J. Bush, Ph. D’Arco, and M. Llunell, CRYSTAL2003, User's Manual (University of Torino, Torino, 2003).

[9] F. Cora, M. Alfredsson, G. Mallia, D. S. Middlemiss, W. C. Mackrodt, R. Dovesi, and R. Orlando, Structure and Bonding, Vol. 113 (Springer-Verlag, Berlin, Heidelberg, 2004), pp. 171-232.

[10] A. D. Becke, J. Chem. Phys. 98, 5648 (1993).

C. Lee, W. Yang, and R. G. Parr, Phys. Rev. B 37, 785 (1988).

[11] J. Heyd and G. E. Scuseria, J. Chem. Phys. 121, 1187 (2004).

[12] D. Muñoz, N. M. Harrison, and F. Illas, Phys. Rev. B 69, 085115 (2004).

[13] W. M. Temmerman, A. Svane, Z. Szotek, and H. Winter, in: Electronic Density Functional Theory: Recent progress and New Directions, edited by J. F. Dobson, G. Vignale, and M. P. Das (Plenum Press, New York, 1998).

[14] P. Blaha, K. Schwarz, G. K. H. Madsen, D. Kvasnicka, and J. Luitz, WIEN2k, An Augmented Plane Wave + Local Orbitals Program for Calculating Crystal Properties, edited by Karlheinz Schwarz (Techn. Universität Wien, Austria, 2001), ISBN 3-9501031-1-2.

[15] J. P. Perdew and A. Zunger, Phys. Rev. B 23, 5048 (1981).

[16] D. J. Singh, Planewaves, Pseudopotentials and the LAPW Method (Kluwer Academic, Dordrecht, 1994).

[17] G. Valerio, M. Cati, R. Dovesi, and R. Orlando, Phys. Rev. B 52, 2422 (1995).

[18] M. D. Towler, N. L. Allan, N. M. Harrison, V. R. Saunders, W. C. Mackrodt, and E. Aprà, Phys. Rev. B 50, 5041 (1994).

[19] M. J. M. de Almeida and P. J. Brown, J. Phys. C 21, 1111 (1988).

[20] V. Fernandez, C. Vettier, F. de Bergevin, C. Giles, and W. Neubeck, Phys. Rev. B 57, 7870 (1998).

[21] B. N. Harmon, V. P. Antropov, A. I. Liechtenstein, I. V. Solovyev, and V. I. Anisimov, J. Phys. Chem. Solids 56, 1521 (1995).

[22] L. W. Rowland, G. J. Cock, F. A. Muller, A. C. Moleman, K. A. M. McEwan, R. L. Jordan, and D. W. Jones, J. Phys. F 5, L233 (1975).

[23] P. Mohn, C. Persson, P. Blaha, K. Schwarz, P. Novak, and H. Eschrig, Phys. Rev. Lett. 87, 196401 (2001). 